\title{
Values Following a Major Terrorist Incident: Finnish Adolescent and Student Values Before and After September 11, 2001
}

\author{
MarkKu Verkasalo ${ }^{1}$ \\ Helsinki University \\ Helsinki, Finland
}

\author{
RobIN GOODwIN \\ Department of Human Sciences \\ Brunel University, London, England
}

\author{
IRINA BEZMENOVA \\ Helsinki University \\ Helsinki, Finland, and Russian State University of Humanities, Moscow, Russia
}

\begin{abstract}
The horrific terrorist attacks on the United States on September 11, 2001, left an indelible mark on perceptions of security and threat across the world. This paper uses Schwartz's (1992) value circumplex model to examine value change across matched high school and university student samples in Finland, questioned before and after the World Trade Center (WTC) and associated attacks. In Study 1 $(N=419)$, security values of adolescents were higher the day following the WTC attacks than before, but fell back toward pre-attack levels in the subsequent two samples. In contrast, levels of stimulation were lower following the terrorist incidents. In Study $2(N=222)$, security levels of students were also higher following the WTC attacks, but again were closer to pre-attack levels in a subsequent cohort.
\end{abstract}

The study of values has reemerged as a major topic for social psychological investigation in the past decade, with value systems seen as an important superordinate cognitive structure with considerable implications for individual behavior (Rohan, 2000; Schwartz \& Bilsky, 1990). A variety of ecological life circumstances - including political structure, historical experiences, and even climatic conditions - has been seen as central to the formation of value priorities (Hofstede, 2001; Schwartz, Bardi, \& Bianchi, 2000), with adaptation and change in value priorities taking place through both the reinforcement contingencies offered by life circumstances and the individual's desire to fulfill outstanding deficit needs (Maslow, 1970; Schwartz \& Bardi, 1997).

${ }^{1}$ Correspondence regarding this article should be addressed to Markku Verkasalo, Department of Psychology, Helsinki University, Helsinki, Finland. E-mail: Markku.Verkasalo @ helsinki.fi

Journal of Applied Social Psychology, 2006, 36, 1, pp. 144-160.

(C) 2006 Copyright the Authors

Journal compilation (c) 2006 Blackwell Publishing, Inc. 
Value change is usually seen as taking place over a relatively long time period, typically decades (De Vos, 1997; Hofstede, 2001), with value stability reinforced by occupational class structures that maintain value consistency across generations (Kohn, Naoi, Schoenbach, Schooler, \& Slomczynski, 1990; Morgan, Alwin, \& Griffin, 1979). Indeed, even when social and political structures undergo radical restructuring, as has been the case over the last decade in Central and Eastern Europe, there has been little evidence of significant value change (Schwartz et al., 2000).

However, what happens to values in the short term, following a major traumatic and public event? The systematic study of the consequences of collective trauma following political violence is a relatively new topic of study (Ladd \& Cairns, 1996; Raviv, Sadeh, Raviv, Silberstein, \& Diver, 2000). Traumatic life events can challenge an individual's assumptive world, undermining implicit but fundamental beliefs and values (Janoff-Bulman, 1989). Clinical studies following terrorist incidents suggest a greater concern for family and personal safety following the witnessing of a terrorist incident (e.g., Applewhite \& Dickins, 1997).

Raviv and colleagues (Raviv, Raviv, Sadeh, \& Silberstein, 1998; Raviv et al., 2000) studied school students' reactions to the assassination of Prime Minister Yitzhak Rabin, both immediately after the murder and 5 months later, as well as responses to a series of terrorist attacks in Israel. Their findings suggest that widespread concerns about the safety of a nation can lead to concerns about physical security, particularly among those with the greatest psychological proximity to the events. Drawing on work by Vertzberger (1997), however, Raviv et al. (2000) suggested that while sudden dramatic events might severely shake an individual's values, "unfreezing" established core beliefs, this may prove to be only a relatively short-lived change. In time, other events in life distract individuals away from the critical terror event; and beliefs, values, and attitudes return to "normal." Thus, as shock and stress wear off, the value pendulum "begins to swing back" (Vertzberger, 1997, p. 870).

The current paper assesses values measured before and after a major terrorist attack, using the value circumplex described by Shalom Schwartz and his associates (e.g., Sagiv \& Schwartz, 1995; Schwartz, 1992). The terrorist event under consideration is the attack on the World Trade Center (WTC) and the associated attacks that took place in the United States on September 11, 2001. In total, the four terrorist attacks on this day killed more than 3,000 people and led to the loss of four civilian aircraft and the destruction of the WTC in New York City and part of the Pentagon building in Washington, DC.

Building on Rokeach's (1973) conceptualization of values, Schwartz et al. (2000) described 10 individual-level value types that satisfy biological needs, interactional requirements, and institutional and social demands for 
group welfare and survival (Schwartz \& Bilsky, 1987). These values are organized in a quasi-circumplex format and can be divided along two dimensions (see Table 1). Dimension 1 compares openness to change (which

Table 1

Values Types

Value $\quad$ Value description

Self-direction Independent thought and action (creativity, freedom, choosing one's own goals, curiosity, independence)

Stimulation Excitement, novelty, challenge in life (an exciting life, varied life, daring)

Conformity Restraint of actions, inclinations, and impulses likely to upset or harm others and violate social expectations/ norms (obedience, self-discipline, politeness, honoring of parents and elders)

Tradition Respect, commitment, and acceptance of customs and ideas that traditional cultures or religion provide (respect for authority, moderation, humility, acceptance of one's own portion in life, devoutness)

Security Safety, harmony, stability of society, of relationships, of self (social order, family security, national security, reciprocation of favors, cleanliness)

Universalism Understanding, appreciation, tolerance, and protection of the welfare of all people and of nature (broadminded, social justice, equality, a world of peace, a world of beauty, unity with nature, wisdom, protection of the environment)

Benevolence Preservation and enhancement of the welfare of people with whom one is in frequent personal contact (loyalty, honesty, helpfulness, responsibility, forgiveness)

Achievement Personal success through demonstrating competence according to social standards (ambition, success, capability, influence)

Power Social status and prestige, dominance over people and resources (social power, wealth, authority)

Hedonism Pleasure and sensuous gratification for oneself (pleasure, enjoyment of life, self-indulgence)

Note. Items in parentheses indicate examples of actual values measured. 
emphasizes independent thought and action and change, and is represented by the values of self-direction and stimulation) with conservation (which stresses submission and self-restriction and includes the values of conformity, tradition, and security). Dimension 2 contrasts a more egalitarian self-transcendence (represented by universalism and benevolence values) with selfenhancement (the values of achievement and power; values that emphasize the pursuit of success and dominance over others). A tenth value, hedonism, is related to both openness to change and self-enhancement (Schwartz, 1994). This model has been tested empirically and verified in more than 70 cultures worldwide, with individual value types significantly correlated with a range of social outcomes, including intergroup perception (Sagiv \& Schwartz, 1995), sexual behavior (Goodwin et al., 2002), and motivation for emigration (Tartakovsky \& Schwartz, 2001).

\section{Research Design and Hypotheses}

The research reported in this paper arose unintentionally from a process of data collection on the values of young people that began in Helsinki in 1998. Research on both political transitions (Inglehart, 1997; Schwartz et al., 2000) and collective trauma (Vertzberger, 1997) suggests that value change is likely to be most marked among the young. Adolescence is a critical period in the formation of values (Bogt, Meeus, Raaijmakers, \& Vollebergh, 2001; Raviv et al., 1998), and senior high school students form the respondents for the first study in this paper.

Our second respondent group, undergraduate students, has formed the subject pool for many of the largest analyses of values across cultures (e.g., Schwartz \& Bardi, 2001), and there is now extensive data on the values of Finnish undergraduates using Schwartz's (1992) value circumplex (Myyry \& Helkama, 2001). These previous data, therefore, provide us with important baseline information for the analysis of value change following the terrorist attacks, as well as a second set of comparative data for the testing of our research hypotheses. In the study reported in this paper, we were able to ascertain exact times that the questionnaires were completed. Therefore, we were able to contrast comparable groups of respondents both before and immediately after the WTC attacks and in the subsequent months following the attacks.

In this study, we drew on the work of Schwartz and Bardi (1997) on social transition, as well as the work of Raviv et al. (1998, 2000) and Vertzberger (1997) on public responses to terrorist attacks to suggest three hypotheses.

Hypothesis 1. The unpredicted, dramatic events that took place in the United States on September 11, 2001, will lead to 
a compensating emphasis on the importance of security values among our samples.

Finland, the country of study in this paper, is a small and relatively secure society, geographically remote from the events of September 11, 2001. As such, our participants were comparatively removed from the long-term consequences of the WTC attacks (Sprang, 1999). In line with Vertzberger's (1997) pendulum hypothesis, we propose the following:

Hypothesis 2. Participants' values will be closer to their preSeptember 11 positions among the later cohorts in our study.

Stimulation values, which emphasize daring and excitement, are the values most directly opposed to security in Schwartz's (1992) circumplex model. Such values emphasize a daring, varied life, which might be expected to correlate with behaviors that involve exposure to enhanced terrorism risk (e.g., going out into potential areas of danger, such as city centers or traveling overseas). Therefore, we propose the following:

Hypothesis 3. Stimulation values will have diminished immediately after the WTC attacks.

Finally, we will examine the moderating influence of gender on value differences before and after the attacks. Girls and women have been shown to report greater threat following traumatic events than boys or men (Norris et al., 2002). Here, we suggest the following:

Hypothesis 4. There will be greater differences in values before versus after the attacks among female respondents.

\section{Study 1: Value Change Among Adolescent School Children}

\section{Method}

\section{Participants}

Respondents were from four samples of senior high school students, one sample that was collected before the WTC attack and three that were collected afterward. Because school type is an important predictor of value scores across a range of societies (Bowles \& Gintis, 1976; Skarzynska, 1993), all respondents were from the Helsinki, Finland, area and attended schools in which entry level was between 8 and 9 (out of a possible 10) on national ability test scores. 
The first sample $(n=301 ; 193$ females, 108 males $)$ was drawn from a larger group of adolescents $(N>500)$ collected during 1998, with the sample used in our study matched for school type with the other samples in this study. Mean age of participants was 16.41 years $(S D=0.51)$.

The second sample ( $n=21 ; 15$ females, 6 males) was collected on the day immediately following the WTC incident (September 12, 2001). Mean age of participants was 17.71 years $(S D=0.64)$.

The third sample ( $n=67 ; 50$ females, 17 males) was collected between 11 and 48 days after the WTC attack (i.e., between September 22, 2001, and November 7, 2001). Mean age of participants was 16.39 years $(S D=0.87)$.

Finally, the fourth sample ( $n=30 ; 24$ females, 6 males) was collected 5 months after the WTC attack (i.e., between February 28, 2002, and March $6,2002)$. Mean age of participants was 17.50 years $(S D=0.68)$.

\section{Measures}

Values were measured using the Schwartz Value Survey (SVS; Schwartz, 1992), a questionnaire previously used in more than 60 countries and with samples including Finnish adolescents (e.g., Verkasalo, Tuomivaara, \& Lindeman, 1996). The back-translated questionnaire includes 57 single values assessed using a 9-point scale, ranging from -1 (opposed to my values) to 7 (of supreme importance). The majority of the values were grouped into the 10 value types described in the introduction of this paper, with the values used in our study listed in Table 1.

To allow for individual variation in scale use, proportional sum variables were used. A personal mean of all 57 values was computed for each respondent separately. Items of the sum variable were then summed and divided by the personal mean multiplied by the number of items included in the sum variable. This gives the proportional importance of any value type compared to other value types, with the mean score for all value items being $1.00{ }^{2}$ Internal reliabilities were similar in magnitude to those reported by Schwartz (1992), and they ranged from .49 (self-direction) to .82 (universalism; $M$ reliability =.72). Questionnaires were distributed as part of a class exercise. Of those attending class, the response rate was in excess of $95 \%$.

\footnotetext{
${ }^{2}$ Unpublished data by the first author show that the method of proportionalization used has a similar effect to partialing the mean. The correlations between direct partialing of the mean method and the proportionalization method varied between .96 and .99 , according to value type.
} 


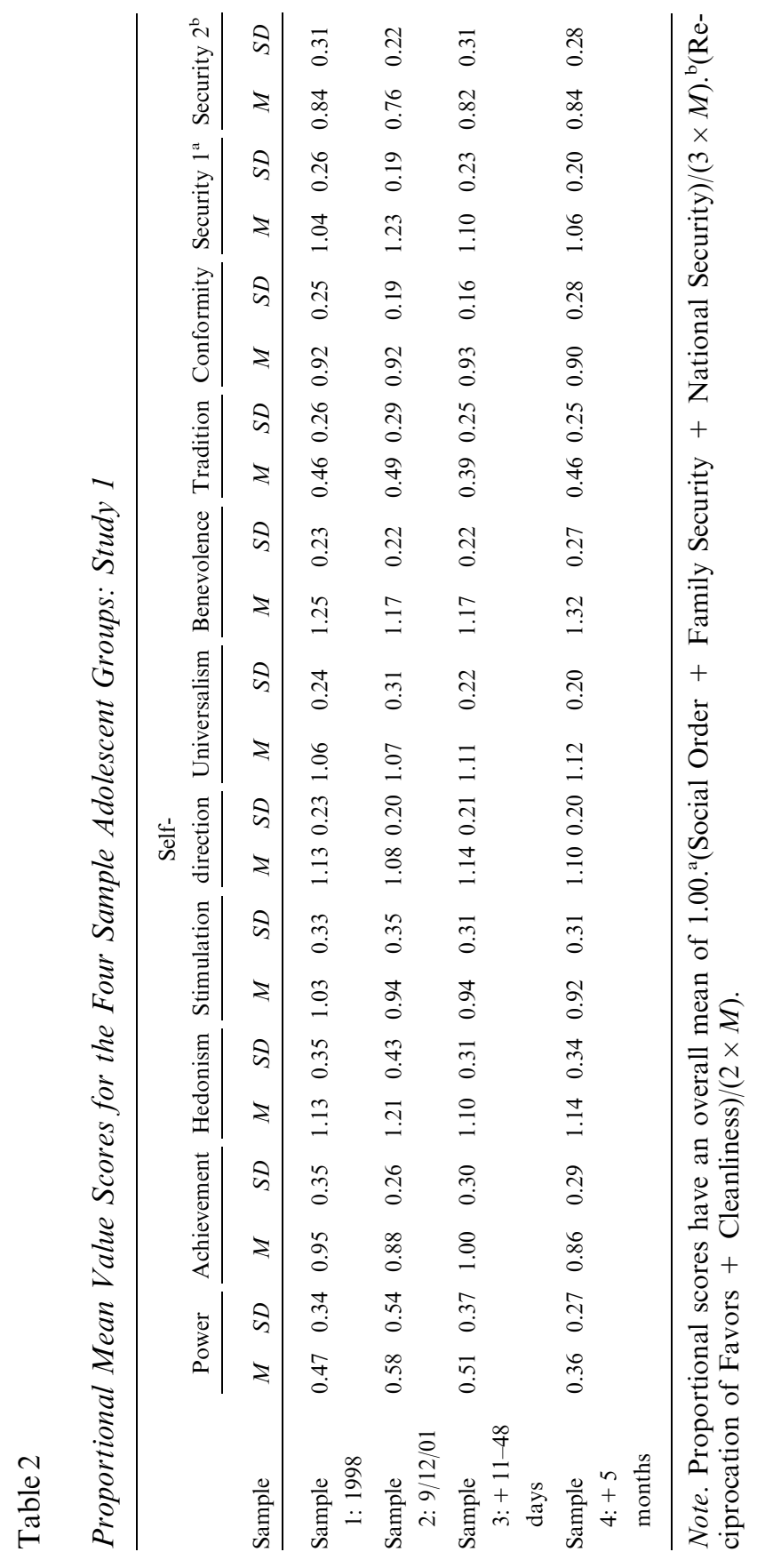




\section{Results}

Table 2 presents proportional mean scores for these 10 values for all samples. Consistent with previous studies investigating Finnish adolescents (Verkasalo et al., 1996), the values of benevolence, universalism, and hedonism were those most highly valued by our samples. The only significant difference between the samples collected in 1998 and those obtained the day after the WTC attack was for security values, with these values significantly higher in the day following the WTC incidents $(M \mathrm{~s}=1.05$ vs. 0.96 , respectively), $t(320)=2.36, p<.05$.

Consistent with the bounce-back hypothesis suggested in this paper's introduction, security values in the later samples were closer to those obtained prior to the WTC attacks ( $M \mathrm{~s}=0.98$ and 0.97 for Samples 3 and 4, respectively). We grouped these security values into two subtypes: individual (reciprocation of favors and cleanliness) and group (social order, family security, and national security), and we report these in Table 2. It was only for the latter subtype that there were significant differences in value scores across the cohorts, $F(7,410)=2.52, p<.02 ; t(320)=3.27, p<.01$, for comparison of before and 1 day after the attacks. As anticipated, stimulation was lower for the samples following the WTC attack, although this difference is significant only for the larger 11-48-day sample, $t(366)=-2.17, p<.04$. Finally, benevolence was also lower for the two immediate post-WTC samples, but higher for the final sample 5 months after the attack, $t(366)=-2.80, p<.01$.

To explore the impact of gender and time following the WTC attacks, we conducted a two-way MANOVA with group-level security and stimulation as dependent variables, and group (before vs. after the WTC attacks) and gender as independent variables. There was no main effect either for gender or for the Gender $\times$ Group interaction, with only group being a significant predictor of value differences, Rao's $F(2,413)=6.20, p=.002$, Wilks's $\lambda=$ .97. Univariate analyses show significant effects for both security and stimulation: security, $F(1,414)=4.37, p=.037$; and stimulation, $F(1$, $414)=9.80, p=.002$. Security scores were higher and stimulation scores were lower following the WTC attacks.

We also conducted a MANOVA on the remaining eight values to see if group had any effect on these values. These analyses demonstrate that neither group, nor the interaction between gender and group had an effect on value differences for the eight values across cohorts.

\section{Discussion}

As hypothesized, adolescent samples collected immediately following the WTC attacks showed significantly higher security scores (when assessed as 
group-level security), and lower levels of stimulation. Also, as expected, subsequent matched samples suggest some evidence of a bounce-back to former security values. Contrary to expectation, benevolence values also demonstrated a U-shaped curve across samples, falling then rising following the WTC attacks.

\section{Study 2: University Students Before and After the Terrorist Attack}

Study 1 was conducted among adolescent high school students, a group previously suggested to be undergoing a developmental period characterized by relatively rapid and widespread value change. Study 2 uses a similar design to examine value change among slightly older groups of participants (i.e., university students) in a second assessment of our hypotheses on value change following the WTC terror events.

\section{Method}

\section{Participants}

The first sample $(n=104 ; 86$ females, 18 males; $M$ age $=26.02$ years, $S D=7.60$ ) was collected from a range of university students at Helsinki University during the year 2000 and in the months before September 2001. The second sample $(n=55 ; 41$ females, 14 males; $M$ age $=23.47$ years, $S D=6.25$ ) was collected in the week following the incident (i.e., September $12-19,2001)$. The third sample $(n=63 ; 47$ females, 16 males; $M$ age $=23.13$ years, $S D=4.59$ ) was collected between 8 and 48 days after the attack (i.e., between September 20, 2001, and November 1, 2001).

\section{Measures}

The same SVS questionnaire (Schwartz, 1992) was employed as was described in Study 1. As before, questionnaires were distributed as part of a class exercise, but this time they were completed online. Internal reliabilities ranged from .47 (tradition) to .80 (power; $M$ reliability $=.67$ ). More than $95 \%$ of those attending class completed the questionnaires.

\section{Results}

Table 3 provides proportionalized mean scores for the values across the three samples. As with the adolescent sample, the values of benevolence, 


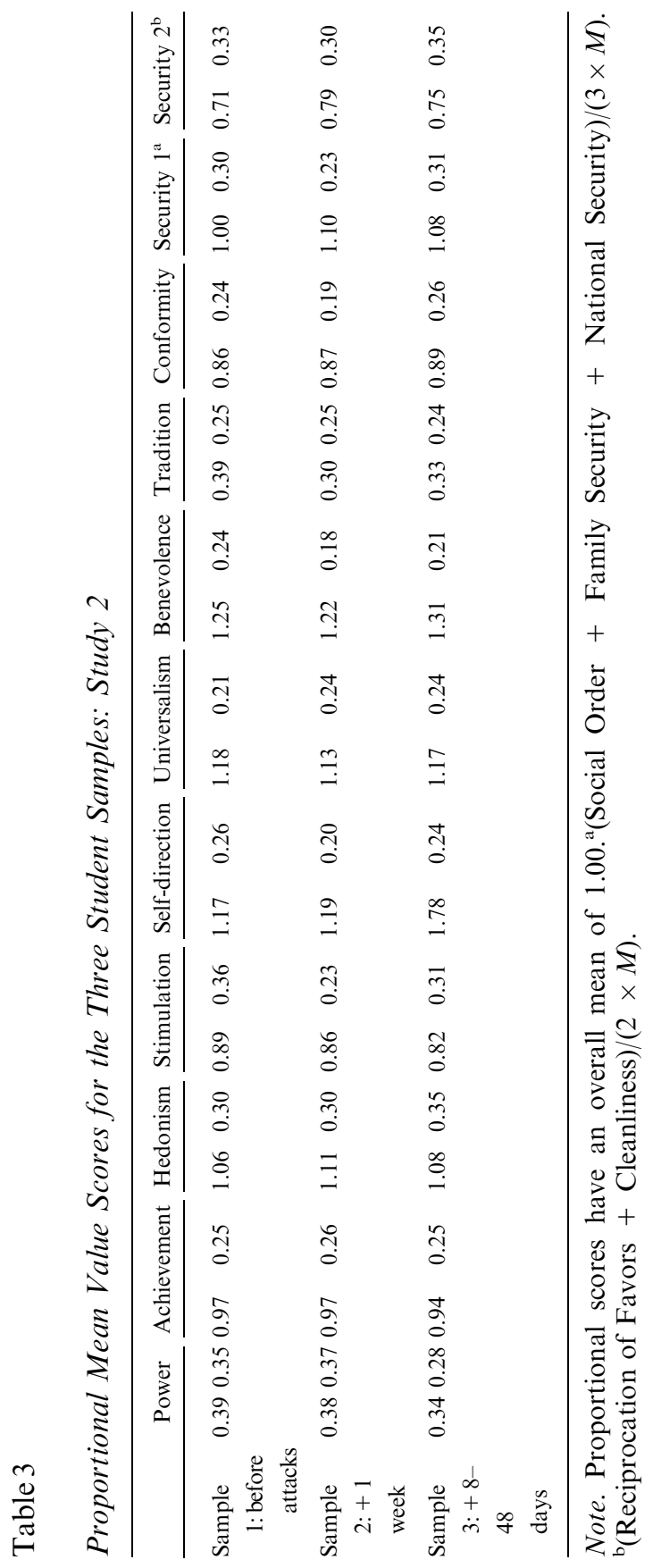


universalism, and hedonism all were evaluated relatively highly by our respondents. However, in common with previous work with this older age group in Finland, self-direction and universalism were also evaluated highly (Verkasalo, Daun, \& Niit, 1994). As in Study 1, security values were significantly higher in the immediate aftermath of the WTC attack $(M \mathrm{~s}=0.97$ vs. 0.89 , respectively), $t(157)=2.46, p<.02$; but were slightly lower in the final data collection $(M=0.94)$. Subdividing security into individual and group subtypes, it was again only group-level security that showed a significant difference across cohorts, $F(5,216)=3.71, p<.01 ; t(157)=2.02, p<$ .03 , for comparison of before and 1 day after the attacks.

Further data trends were also similar to those reported in Study 1, but here the differences across samples were not significant. Stimulation scores were lower in the samples following the WTC attacks, while scores in benevolence were first lower then higher in the samples collected after the terrorist attacks. Finally, tradition scores were significantly lower in the sample taken immediately after the WTC attack, $t(157)=-2.18, p<.05$, for Samples 1 and 2, but were marginally higher again in the final sample.

We repeated our 2 (Group) $\times 2$ (Gender) MANOVA as in Study 1, with group-level security and stimulation as the dependent variables. There was no main effect either for gender or for the Gender $\times$ Group interaction, with only group being a significant predictor of value differences, Rao's $F(2$, $217)=3.36, p=.04$, Wilks's $\lambda=.97$. Univariate analyses show significant effects for security, $F(1,218)=4.77, p=.030$; but there was no significant effect for stimulation.

We also conducted a MANOVA on the remaining eight values to see if group had any effect on these values. These analyses demonstrate that neither group nor the interaction between gender and group had an effect on value differences for the eight values across cohorts.

\section{Discussion}

This student study also demonstrated higher group-level security values following the WTC attacks, followed by a return to value scores closer to those previous to the attacks. Changes in stimulation and benevolence also demonstrated similar trends to the patterns obtained in Study 1. In addition, in Study 2, tradition showed a significant drop following the WTC attack, although this effect was not maintained in our final data collection.

In our paper's introduction, we suggested that adolescence is a critical period in the formation of values (Bogt et al., 2001; Raviv et al., 1998). Therefore, we were interested to see if value differences following the WTC attacks were greater among our adolescents, compared to our student sam- 
ples. To do this, we conducted a three-way MANOVA, with study group (adolescents vs. students), gender, and group (before vs. after the WTC attacks) as our independent variables, and group-level security and stimulation as the dependent variables. To ensure comparability between study groups and sufficient cell frequency size, we did not include the adolescent data collected 5 months after the WTC attacks. The three-way interaction was not statistically significant, nor were the interactions of Group $\times$ Study Group or Group $\times$ Gender. This suggests that value differences are no larger for students or adolescents (or for male vs. female participants) in our studies.

As expected, group had a significant main effect, $F(2,602)=8.45, p<$ .0003 , Wilks's $\Lambda=.97$. A univariate analysis for the group variable shows that both security $(p=.0004)$ and stimulation $(p=.005)$ were statistically different between the before- and after-attack conditions, with security values higher and stimulation values lower. Study group itself also had an effect (Wilks's $\Lambda=.98$ ), Rao's $F(2,602)=7.20, p=.000043$, with both stimulation and security averages higher for adolescents than for students. There was also an interaction between gender and study group (adolescents vs. students; Wilks's $\Lambda=.98)$, Rao's $F(2,602)=4.10, p=.02$. For university females, security was as high as for adolescent females, while stimulation was especially low for university females.

We also ran three-way MANOVAs for the values for which we did not have special hypotheses. The three-way interaction was not significant; neither were any of the two-way interactions (i.e., Study Group $\times$ Group, Study Group $\times$ Gender, or Group $\times$ Gender). Instead, study group itself was significant, Rao's $F(9,595)=4.44, p<.001$ (Wilks's $\Lambda=.94$ ). The univariate tests (using Bonferroni's post hoc correction) show that security (individual level), power, and tradition were higher for adolescents; and that self-direction and universalism were higher for students. There also were significant gender effects, Rao's $F(9,595)=6.46, p<.001$, (Wilks's $\Lambda=.91$ ), with univariate tests showing power and self-direction to be higher for males; while universalism and benevolence were higher for female participants.

\section{General Discussion}

The terrorist attacks on the United States on September 11, 2001, left an indelible mark on perceptions of security and threat across the world. In the data reported in this paper, samples of adolescents and university students in Finland demonstrated significantly higher group-level security values immediately following these attacks, although there were signs of a return to previous levels of security value scores in later samples. These differences 
were evident, irrespective of gender or participant group (university student or adolescent).

In addition, trend data in both studies suggest a drop in the opposing value of stimulation, and a U-shaped decline and then rise in benevolence in the period following these terrorist incidents. These observed differences in security scores following the terrorist attacks are consistent with previous research on terrorist events reviewed in this paper's introduction. Such findings are also in line with the conclusions of Boehnke's (2001) review of intergenerational value transmission, which suggests that security values are among those most likely to be influenced by the immediate social context (what he terms the zeitgeist effect).

Security values involve perceptions of both basic individual and group security (Schwartz, 1992). In both studies, it was group-level security values (i.e., values of social order, family security, and national security) that were different across the cohorts, with levels of security particularly high among our adolescent samples. At the group level, societies high in insecurity demonstrate a need for order and demonstrate little tolerance for unpredictability (Inglehart, 1997). Such societies demand strong leaders, and may promote a turn toward higher religious authorities for spiritual guidance during times of uncertainty and change (Inglehart, 1997). For all this, of course, our data suggest that, after a relatively short period of time, other life distractions, perhaps coupled with a perceived lessening of personal threat, can lead to a bounce-back toward former value positions (Vertzberger, 1997). We suspect, however, that the extent of this bounceback is likely to be dependent on the degree to which the perceived threat remains (Janoff-Bulman, 1989). Such findings, we believe, reinforce the ecological position that the long-term consequences of any value change are likely to be dictated largely by the actual day-to-day patterns of living in any one society (Schwartz \& Bardi, 1997).

Other trends in our data were predicted only partially. Perhaps unsurprisingly, stimulation reflects a desire for excitement strongest among our adolescent samples. Stimulation is the value most directly opposed to security in Schwartz's (1992) circumplex model, and the lower scores on this value type following the WTC were anticipated. This decline in the desire for an exciting and challenging life may reflect what many refer to as a new "cautiousness" about traveling, evident worldwide in the months following the WTC attacks. Furthermore, while security values might have bounced back relatively quickly when it became evident that Finland was not a likely terrorism target, wider international precautions (e.g., new airport regulations, flight cancellations) might have helped to maintain the decline in stimulation values.

The U-shaped effect for benevolence scores, although significant only in Study 1, was intriguing, however. Benevolence in Schwartz's (1992) model 
concerns the "preservation and enhancement of the welfare of people with whom one is in frequent personal contact" (Schwartz, 1994, p. 22); and previous studies have suggested the development of relatively strong, benevolent ties following terror attacks (Vertzberger, 1997).

One explanation for our findings, of course, may be the physical and psychological distance of the WTC attacks from our Finnish respondents. It is unlikely, for example, that many members of our young samples knew anyone who was affected by the attacks directly. Therefore, the motivation to form such beneficent networks may have been lower.

A second interpretation for our benevolence findings focuses on the processes likely to lead to value change. Theorists of cultural change have identified three channels of value transmission: vertical (e.g., parent-child), horizontal (e.g., peer-peer), and oblique (e.g., via the media; Schönpflug, 2001a). As media-viewing figures and newspaper sales clearly demonstrate, the WTC and associated attacks were overwhelming media events that led, in Finland at least, to a fairly universal response of shock and horror. The predominance of this medium during this time, coupled with the lack of significant subgroups that questioned the motivations behind and implications of this attack, meant that there was neither the opportunity nor the motivation to develop the collectivistic, in-group ties characteristic of those holding high benevolence values.

Over time, of course, other forms of value transmission may be of significance when assessing the full impact of the terrorist attacks of September 2001. Here, we might anticipate a greater horizontal interaction among young respondents considering responses to the terror events (cf. Schönpflug, 2001a, on value transmission between peer groups), while at the same time, collectivistic values (e.g., group-level security) may be reinforced via vertical, intergenerational exchanges (Cavalli-Sforza \& Feldman, 1981; Schönpflug, 2001b).

Interestingly, neither age nor gender seemed to modify the extent to which values varied before versus after the WTC attacks, although study group and Study Group $\times$ Gender interactions were significant for some of the values. However, the strong female bias in our samples, and the relatively small age differences between our adolescent and student samples, rather restricted the power of our analyses. Future research should investigate such potentially moderating factors with wider and more representative samples.

Understanding responses to terror attacks is an important topic for social psychological investigation, presenting social researchers with a significant opportunity to study some of the most fundamental aspects of individual stability and change. However, such a topic has been largely neglected by social psychologists, despite the (unfortunately increasing) opportunities for 
such investigations (Raviv et al., 2000). The present study suggests that even within a relatively "remote" culture such as Finland, large-scale terrorist attacks may have a significant impact on individual values. At the same time, of course, the current study suffers from a number of limitations.

Our work can only hope to approximate value change and did not examine value change per se, as each value assessment was independent. In Study 1, our first wave of data was collected in 1998, which was 3 years before the WTC attacks. Although other unpublished data have suggested little change in values between 1991 and 1997, more proximal data would have been desirable.

Work on post-traumatic stress suggests that a wide range of sociodemographic factors (e.g., socioeconomic background) might influence responses to threat (Norris et al., 2002), and the moderating effects of such variables should be considered in future investigations. Therefore, future studies should continue this work, using genuine longitudinal data to investigate the impact of terror attacks on a range of social groups across a variety of affected cultures.

\section{References}

Applewhite, L., \& Dickins, C. (1997). Coping with terrorism: The OPMSANG experience. Military Medicine, 162, 240-243.

Boehnke, K. (2001). Parent-offspring value transmission in a societal context. Journal of Cross-Cultural Psychology, 32, 241-255.

Bogt, T. F. M., Meeus, W. H. J., Raaijmakers, Q. A. W., \& Vollebergh, W. A. M. (2001). Youth centrism and the formation of political orientations in adolescence and young adulthood. Journal of Cross-Cultural Psychology, 32, 229-240.

Bowles, S., \& Gintis, H. (1976). Schooling in capitalist America: Educations reform and the contradictions of economic life. New York: Basic Books.

Cavalli-Sforza, L. L., \& Feldman, M. W. (1981). Cultural transmission and evolution: A quantitative approach. Princeton, NJ: Princeton University Press.

De Vos, G. (1997). Heritage of exploitation: A brief TAT report on South Brazilian youth. Political Psychology, 18, 439-481.

Goodwin, R., Realo, A., Kwiatkowska, A., Kozlova, A., Nguyen-Luu, L. A., \& Nizharadze, G. (2002). Values and sexual behavior in Central and Eastern Europe. Journal of Health Psychology, 7, 45-56.

Hofstede, G. (2001). Culture's consequences: Comparing values, behaviors, organizations across nations. (2nd ed.). London: Sage. 
Inglehart, R. (1997). Modernization and postmodernization: Cultural, economic, and political change in 43 countries. Princeton, NJ: Princeton University Press.

Janoff-Bulman, R. (1989). Assumptive worlds and the stress of traumatic events: Applications of the schema construct. Social Cognition, 7, 113-136.

Kohn, M. L., Naoi, A., Schoenbach, C., Schooler, C., \& Slomczynski, K. M. (1990). Position in the class structure and psychological functioning in the United States, Japan, and Poland. American Journal of Sociology, 95, 964-1008.

Ladd, G. W., \& Cairns, E. (1996). Children: Ethnic and political violence. Child Development, 67, 14-18.

Maslow, A. (1970). Motivation and personality. (2nd ed.). New York: Harper \& Row.

Morgan, W. R., Alwin, D. F., \& Griffin, L. J. (1979). Social origin, parental values, and the transmission of inequality. American Journal of Sociology, 85, 156-166.

Myyry, L., \& Helkama, K. (2001). University students' value priorities and emotional empathy. Educational Psychology, 21, 25-40.

Norris, F. H., Friedman, M. J., Watson, P. J., Byrne, C. M., Diaz, E., \& Kaniasty, K. (2002). 60,0000 disaster victims speak. Part I: An empirical review of the empirical literature, 1981-2001. Psychiatry, 65, 207-239.

Raviv, A., Raviv, A., Sadeh, A., \& Silberstein, O. (1998). The reaction of the youth in Israel to the assassination of Prime Minister Yitzhak Rabin. Political Psychology, 19, 255-278.

Raviv, A., Sadeh, A., Raviv, A., Silberstein, O., \& Diver, O. (2000). Young Israeli's reactions to national trauma: The Rabin assassination and terror attacks. Political Psychology, 21, 299-322.

Rohan, M. (2000). A rose by any name? The values construct. Personality and Social Psychology Review, 4, 255-277.

Rokeach, M. (1973). The nature of human values. New York: Free Press.

Sagiv, L., \& Schwartz, S. H. (1995). Value priorities and readiness for outgroup social contact. Journal of Personality and Social Psychology, 69, 437-448.

Schönpflug, U. (2001a). Cultural transmission: A multidisciplinary research field. Journal of Cross-Cultural Psychology, 32, 131-134.

Schönpflug, U. (2001b). Intergenerational transmission of values: The role of transmission belts. Journal of Cross-Cultural Psychology, 32, 174-185.

Schwartz, S. (1992). Universals in the content and structure of values: Theoretical advances and empirical tests in 20 countries. In M. P. Zanna (Ed.), Advances in experimental social psychology (Vol. 25, pp. 1-65). New York: Academic Press. 
Schwartz, S. H. (1999, June 28-July 3). Cultural dimensions and value types. Workshop presented at conference of the International Association for Cross-Cultural Psychology, Graz, Austria.

Schwartz, S. H., \& Bardi, A. (1997). Influences of adaptation to Communist rule on value priorities in Eastern Europe. Political Psychology, 18, $385-410$.

Schwartz, S. H., \& Bardi, A. (2001). Value hierarchies across cultures: Taking a similarities perspective. Journal of Cross-Cultural Psychology, 32, 268-290.

Schwartz, S. H., Bardi, A., \& Bianchi, G. (2000). Value adaptation to the imposition and collapse of Communist regimes in Eastern Europe. In S. A. Renshon \& J. Duckitt (Eds.), Political psychology: Cultural and cross-cultural perspectives (pp. 217-237). London: Macmillan.

Schwartz, S. H., \& Bilsky, W. (1987). Toward a universal psychological structure of human values. Journal of Personality and Social Psychology, $53,550-562$.

Schwartz, S. H., \& Bilsky, W. (1990). Toward a theory of the universal content and structure of values: Extensions and cross-cultural replications. Journal of Personality and Social Psychology, 58, 878-891.

Skarzynska, K. (1993). Determinants of values accepted by youth. Polish Psychological Bulletin, 24, 195-207.

Sprang, G. (1999). Post-disaster stress following the Oklahoma City bombing: An examination of three community groups. Journal of Interpersonal Violence, 14, 169-183.

Tartakovsky, E., \& Schwartz, S. H. (2001). Motivation for emigration, values, well-being, and identification among young Russian Jews. International Journal of Psychology, 36, 88-99.

Verkasalo, M., Daun, A., \& Niit, T. (1994). Universal values in Estonia, Finland, and Sweden. Ethnologia Europaea, 24, 101-117.

Verkasalo, M., Tuomivaara, P., \& Lindeman, M. (1996). 15-year-old pupils' and their teachers' values, and their beliefs about the values of an ideal pupil. Educational Psychology, 16, 35-47.

Vertzberger, Y. Y. I. (1997). The antimonies of collective political trauma: A pre-theory. Political Psychology, 18, 863-876. 